\title{
Stock Price Jump-diffusion Process Model Based on Fractional Brownian Motion Theory
}

\author{
Hui Ma \\ Jilin Agricultural Science and Technology University \\ Jilin, 132101, China
}

\author{
Yang Li \\ Jilin Agricultural Science and Technology University \\ Jilin, 132101, China
}

\begin{abstract}
In order to deal with the sudden change of stock price and simplify the empirical research, the jump-diffusion process factor of the fractional Brownian motion jump-diffusion process model is modified. The jump diffusion process item is approximated by the polynomial, and the numerical algorithm simulates the real stock price change. The model has fast convergence speed and high precision.
\end{abstract}

Keywords-Stock price; Fractional Brownian motion; Jump-diffusion process; Numerical simulation

\section{INTRODUCTION}

At present, the research on the stock price theory mainly includes two aspects. First, based on the non-linear model, the mixed theory and the fractal theory, the stock price is studied by using the neural network and the fuzzy system. Second, based on the theory of fractional Brownian motion, the stock price is studied by means of fractal properties. Fractal Brownian motion, first proposed by B. B. Mandelbrot and Van Ness, is used to simulate various kinds of noises with fractal characteristics. It has become a mathematical model that can reflect the natural object's properties of a wide range. Edgar Peters put forward the fractal market hypothesis. The fractal market hypothesis emphasizes the influence of liquidity and the starting point of investment based on investors' behavior. Its purpose is to give a model of investors' behavior and market price movement in line with investors' expectations [1]. Peters analyzes different capital markets with R/S analysis method, such as stock market return and exchange rate, and finds fractal structure and aperiodic cycle, which proves that capital market is nonlinear system [2]. Cipiran. $\mathrm{N}$ has established the fractional B-S formula based on the fractional Brownian motion model. McCulloch has proposed the option pricing formula under the stable distribution. However, Decius fund and Ustunnel have developed the option pricing formula based on the fractal Brownian motion by using path dependent integral, thus laying the foundation for the warrant pricing under the fractal market hypothesis [3]. Duncan, $\mathrm{Hu}$ and Pasik Duncan deduce that Hurst index belongs to $(0.5,1)$ fractal Brownian motion integral based on wick operator, and prove that the market is arbitrage free under the hypothesis of fractal Brownian motion. Thus, the pricing model of European call options under fractal Brownian motion is derived. Beckera $\mathrm{S}$ effectively estimates the parameters of this jump-diffusion process model [4]. Yu Zuowei et al. studied the pricing model of convertible bonds under mixed fraction Brownian motion. Based on the market equilibrium condition, the average risk neutral measure was obtained. By using the risk-neutral pricing

The "13th five-year" science and technology project of the Education Department of Jilin Province (No. JJKH20170338KJ). principle, the pricing formula of convertible bonds and the explicit expression of the derivative on Hurst parameter are obtained [5]. Lin Hanyan studies the pricing of American two value options under fractional Brownian motion; the pricing problem is divided into two parts: the price of a permanent American option and the solution of a Cauchy problem, and the pricing formula are obtained [6].

Based on these basic theoretical models and empirical research, the stock price pricing model has made great progress. However, in the process of option pricing, especially in the simulation of stock price change, market friction and distortion produce a "smile curve" of stock price volatility. To deal with such problems, more random factors should be introduced into the model, such as jump-diffusion process, random volatility model, etc., which can better reflect the actual situation of stock price change. Based on the jump diffusion process of fractional Brownian motion, the expected return $(\mu)$ and volatility $(\sigma)$ of stock are constructed as functions of time (t, i.e., $\mu(\mathrm{t})$ and $\sigma(\mathrm{t}))$ and a sum of continuous non-random functions (i.e., $\mu(\mathrm{t}, \mathrm{S}(\mathrm{t}))$ and $\sigma(\mathrm{t}, \mathrm{S}(\mathrm{t})))$. The jump-diffusion process term is modified in two directions, and the scaling factor and generalized Poisson compensation process are introduced. At the same time, the polynomial is used to approximate the jump-diffusion process term for numerical simulation.

The models and algorithms proposed in this work are based on the following basic assumptions.

(1) Capital market is a fractal market.

(2) Risk-free interest rates are constant.

(3) There is no dividend on the stock (i.e., the expected rate of return is the bank interest rate).

(4) There are no transaction costs and taxes.

(5) The stock price is a fractal time series in the continuous trading day.

(6) In addition to continuous changes, there is also a sharp jump caused by some special social, political or psychological factors.

(7) The stock price data are really reliable and are a continuous time series.

\section{MOdEl ANAlysis}

The classical fractional jump-diffusion process model: 


$$
d S(t)=\mu S(t)+\sigma S(t)\left(d B_{H}(t)+d Q(t)\right)
$$

Its discrete form:

$$
\mathrm{S}\left(\mathrm{t}_{\mathrm{k}+1}\right)=\mathrm{S}\left(\mathrm{t}_{\mathrm{k}}\right)+\mathrm{S}\left(\mathrm{t}_{\mathrm{k}}\right)\left[\mu \Delta \mathrm{t}+\sigma\left(\mathrm{W}_{\mathrm{H}}\left(\mathrm{t}_{\mathrm{k}}\right)+\Delta \mathrm{Q}\left(\mathrm{t}_{\mathrm{k}}\right)\right)\right] .
$$

The fractional jump-diffusion process model is modified as follows. The expected return $(\mu)$ and volatility $(\sigma)$ of stock are constructed as functions of time (t, i.e., $\mu(t)$ and $\sigma(t))$, which makes the model increase the influence of the time factor on expected return rate and volatility. And in order to better control the intensity of jump, the jump variable (dQt), is modified to introduce the scaling factor $\left(\mathrm{U}_{\mathrm{t}}\right)$, and the jump term is modified to $\left(\mathrm{e}^{\mathrm{U}_{\mathrm{t}}}-1\right) \mathrm{dQ}(\mathrm{t})$ [7].

The model is established as follows:

$$
\mathrm{dS}(\mathrm{t})=\mu(\mathrm{t}) \mathrm{S}(\mathrm{t}) \mathrm{dt}+\sigma \mathrm{S}(\mathrm{t})\left[\mathrm{dB}_{\mathrm{H}}(\mathrm{t})+\left(\mathrm{e}^{\mathrm{U}_{\mathrm{t}}}-1\right) \mathrm{dQ}(\mathrm{t})\right] .
$$

Recorded as model 1. Among them, $U_{t}$ is an independent uniform distribution sequence, which obeys the uniform distribution on $(-\mathrm{a}, \mathrm{a})$. In particular, when $\mathrm{a}=0$, it is the ordinary geometric fraction Brownian motion, and the model is more general.

The discrete form can be expressed as follows:

$$
\begin{aligned}
\mathrm{S}\left(\mathrm{t}_{\mathrm{k}+1}\right)=\mathrm{S}\left(\mathrm{t}_{\mathrm{k}}\right) & +\mathrm{S}\left(\mathrm{t}_{\mathrm{k}}\right)\left[\mu\left(\mathrm{t}_{\mathrm{k}}\right) \Delta \mathrm{t}+\sigma\left(\mathrm{t}_{\mathrm{k}}\right) \mathrm{W}_{\mathrm{H}}\left(\mathrm{t}_{\mathrm{k}}\right)\right. \\
& +\left(\mathrm{e}^{\left.\left.\mathrm{U}_{\mathrm{t}_{\mathrm{k}}}-1\right) \Delta \mathrm{Q}\left(\mathrm{t}_{\mathrm{k}}\right)\right]}\right.
\end{aligned}
$$

$\mathrm{W}_{\mathrm{H}}\left(\mathrm{t}_{\mathrm{k}}\right)$ represents the increment of fractional Brownian motion (i.e., fractional white noise). When $\left(e^{U_{t}}-1\right) d Q(t)$ is used as jumping term to control jumping intensity, it may appear relatively stable state after short-term sharp jumping, which is not in line with the reality of stock market, and the calculation amount of large-scale numerical simulation is large. For the convenience of numerical simulation and simplification of the algorithm, the Taylor expansion of $\mathrm{e}^{\mathrm{U}_{\mathrm{t}}}$ is used to approximate it as a low degree polynomial:

$$
\mathrm{e}^{\mathrm{U}_{\mathrm{t}}}=\sum_{\mathrm{k}=1}^{\mathrm{n}} \frac{1}{\mathrm{k} !} \mathrm{U}_{\mathrm{t}}^{\mathrm{m}},
$$

The order (n) of the expansion term is adjusted according to the actual situation, usually taking $n=1,2$, 3 . For example, when $\mathrm{n}=1$, it can be expressed as:

$$
\begin{aligned}
S\left(t_{k+1}\right)=S\left(t_{k}\right) & +S\left(t_{k}\right)\left[\mu\left(t_{k}\right) \Delta t+\sigma\left(t_{k}\right) W_{H}\left(t_{k}\right)\right. \\
& \left.+\left(U\left(t_{k}\right)+U\left(t_{k}\right)^{2} / 2\right) \Delta Q\left(t_{k}\right)\right],
\end{aligned}
$$

In terms of long-term trend, the frequency of stock jump is low. However, model 1 can't reflect the time extensibility of jump form. The jump form in the fractional jump diffusion process model is introduced into the generalized compensated Poisson process, which reflects the temporal extensibility of the jump form. The model 1 is modified to a process that can control the jump frequency. The expected return $(\mu)$ and volatility $(\sigma)$ of stock are further improved to continuous nonrandom functions (i.e., $\mu(\mathrm{t}, \mathrm{S}(\mathrm{t}))$ and $\sigma(\mathrm{t}, \mathrm{S}(\mathrm{t}))$ ) of time and stock price.

The model is established as follows:

$$
\begin{gathered}
d S(t)=S(t)\left[\mu(t, S(t)) d t+\sigma(t, S(t)) d B_{H}(t)\right. \\
\left.+\left(e^{U_{t}}-1\right) d N(t)\right] .
\end{gathered}
$$

Its discrete form:

$$
\begin{aligned}
S\left(t_{k+1}\right)=S\left(t_{k}\right)+ & S\left(t_{k}\right)\left[\mu\left(t_{k}, S\left(t_{k}\right)\right) \Delta t\right. \\
& +\sigma\left(t_{k}\right) W_{H}\left(t_{k}, S\left(t_{k}\right)\right) W_{H}\left(t_{k}\right) \\
& \left.+\left(e^{U\left(t_{k}\right)}-1\right) \Delta N\left(t_{k}\right)\right] .
\end{aligned}
$$

Recorded as model 2. Among them, $\{\mathrm{N}(\mathrm{t}), \mathrm{t} \geq 0\}$ is a generalized compensated Poisson process (i.e., usually taking $0<m \leq \frac{1}{2}$ ). In particular, when $m=1$, this model is a fractional jump-diffusion model for Poisson process with compensation, and the model is more general.

For the convenience of calculation, $\mathrm{e}^{\mathrm{U}_{\mathrm{t}_{\mathrm{k}}}}$ can be expanded in the form of the above polynomials, such as $n=2$, which can be expressed as:

$$
\begin{gathered}
\mathrm{S}\left(\mathrm{t}_{\mathrm{k}+1}\right)=\mathrm{S}\left(\mathrm{t}_{\mathrm{k}}\right)+\mathrm{S}\left(\mathrm{t}_{\mathrm{k}}\right)\left[\mu\left(\mathrm{t}_{\mathrm{k}}\right) \Delta \mathrm{t}+\sigma\left(\mathrm{t}_{\mathrm{k}}\right) \mathrm{W}_{\mathrm{H}}\left(\mathrm{t}_{\mathrm{k}}\right) \mathrm{W}_{\mathrm{H}}\left(\mathrm{t}_{\mathrm{k}}\right)\right. \\
\left.+\left(\mathrm{U}\left(\mathrm{t}_{\mathrm{k}}\right)+\mathrm{U}\left(\mathrm{t}_{\mathrm{k}}\right)^{2} / 2\right) \Delta \mathrm{N}_{\mathrm{k}}\right] .
\end{gathered}
$$

\section{NUMERICAL ALGORITHM}

The numerical simulation algorithm is used to realize the model. For model 1 and model 2, the following numerical algorithms are used in this work.

For fractional Brownian motion, a fast simulation algorithm that takes into accounts both efficiency and accuracy proposed by Yin Z-M [8] is used to deal with the increment of fractional Brownian motion.

The increment of fractional Brownian motion is defined:

$$
\mathrm{W}_{\mathrm{H}(\mathrm{t})}=\mathrm{B}_{\mathrm{H}}(\mathrm{t}+\Delta \mathrm{t})-\mathrm{B}_{\mathrm{H}}(\mathrm{t}), \mathrm{t} \geq 0 .
$$

Among them, $\Delta t$ is the change of time. Since fractional Brownian motion $\left(\mathrm{B}_{\mathrm{H}}(\mathrm{t})\right)$ is a continuous Gaussian process, $\mathrm{W}_{\mathrm{H}(\mathrm{t})} \sim \mathrm{N}\left(0, \Delta \mathrm{t}^{2 \mathrm{H}}\right)$ are called fractional white noise. When $\mathrm{H}=\frac{1}{2}, \mathrm{~W}_{\mathrm{H}(\mathrm{t})}$ becomes ordinary white noise. By simulating the fractional white noise, the fractional Brownian motion $\left(\mathrm{B}_{\mathrm{H}}(\mathrm{t})\right)$ can be obtained by adding the increment of the fractional white noise $\left(\mathrm{W}_{\mathrm{H}(\mathrm{t})}\right)$.

The autocorrelation function of $\mathrm{W}_{\mathrm{H}(\mathrm{t})}$ is obtained from the properties of fractional Brownian motion:

$$
\mathrm{C}(\mathrm{r})=\frac{1}{2}\left(|\mathrm{r}+1|^{2 \mathrm{H}}+|\mathrm{r}-1|^{2 \mathrm{H}}-2|\mathrm{r}|^{2 \mathrm{H}}\right), \mathrm{r}=0, \pm 1, \pm 2 \cdots .
$$

Power spectral density function:

$$
f(\lambda)=\sum_{r=-\infty}^{+\infty} C(r) \cos 2 \pi r \lambda,-\frac{1}{2} \leq \lambda \leq \frac{1}{2} .
$$

The autocorrelation function $(\mathrm{C}(\mathrm{r}))$ can be represented by the inverse Fourier transform of $f(\lambda)$ :

$$
C(r)=\int_{\frac{1}{-2}}^{\frac{1}{2}} f(\lambda) \cos (2 \pi r \lambda) d \lambda,-\frac{1}{2} \leq \lambda \leq \frac{1}{2} .
$$


The spectral density function and correlation function of stationary process can be expressed according to Fourier transform. The corresponding process is generated by spectral method:

$$
\begin{gathered}
\mathrm{W}_{\mathrm{H}}(\mathrm{t})=\sqrt{2} \sum_{\mathrm{k}=-\frac{\mathrm{N}}{2}}^{\frac{\mathrm{N}}{2}}\left[\mathrm{f}\left(\lambda_{\mathrm{k}}\right) \Delta \lambda\right]^{\frac{1}{2}} \cos 2 \pi \lambda_{\mathrm{k}} \mathrm{t}+\phi_{\mathrm{k}}, \\
\mathrm{t}=0,1,2, \cdots, \mathrm{N}-1 .
\end{gathered}
$$

Among them, $N$ is the number of samples taken on $f$, and $\Delta \lambda=1 / \mathrm{N}$ is the sample interval. $\lambda_{\mathrm{k}}=\mathrm{k} \Delta \lambda$ is the sample value of $\mathrm{f}$, and $\phi_{\mathrm{k}}$ is the independent random angle of uniform distribution on $[0,2 \pi]$. From the self-similarity of $\mathrm{B}_{\mathrm{H}}(\mathrm{t}),(\mathrm{T} / \mathrm{H})^{\mathrm{H}} \mathrm{W}_{\mathrm{H}(\mathrm{t})}$ is the increment of $\mathrm{B}_{\mathrm{H}}(\mathrm{t})$ on time[0,T], and then $B_{H}(t)$ can be generated by accumulation.

The generalized compensation Poisson process $(\mathrm{N}(\mathrm{t})=$ $\left.\mathrm{Q}(\mathrm{t})-\lambda \mathrm{t}^{\mathrm{m}}, 0 \leq \mathrm{m} \leq 1\right)$ is simulated by MATLAB. A Poisson process and a corresponding increment are first generated. The orbit of Poisson process is a monotone jump function, and the adjacent jump intervals are independent of the exponential distribution. Thus, the sample function of the Poisson process can be simulated using the following steps:

(1) A series of random numbers uniformly distributed and independent of each other on $[0,1]$ is generated, which is recorded as $\left\{\mathrm{U}_{\mathrm{n}}, \mathrm{n} \geq 1\right\}$.

(2) Letting $X_{k}=-\lambda^{-1} \ln U_{k}$ be a known parameter, it is easy to prove that $\left\{X_{n}, n \geq 1\right\}$ is an independent random variable with the same exponential distribution, and setting the

(3) $. \mathrm{S}_{0}=0, \mathrm{~S}_{\mathrm{n}}=\sum_{\mathrm{k}=1}^{\mathrm{n}} \mathrm{X}_{\mathrm{k}}$.

(4) Definition $\mathrm{N}(\mathrm{t})$ is as follows: if $0 \leq \mathrm{t} \leq \mathrm{S}_{1}$, then $\mathrm{N}(\mathrm{t})=0$. If $\mathrm{S}_{\mathrm{n}} \leq \mathrm{t} \leq \mathrm{S}_{\mathrm{n}+1}$, then $\mathrm{N}(\mathrm{t})=\mathrm{n}$. If this continues, one of $\{\mathrm{N}(\mathrm{t}), \mathrm{t} \geq 0\}$ orbits will be obtained.

\section{NUMERICAL SIMULATIONS}

The Hurst index of the stock is calculated by the R / S analysis method (i.e., the rescaled range method). Through this method, the Hurst index with the stock code of 601318 (Ping An, China) is analyzed. The closing price of 1144 trading days is taken as the original data. The calculation of Hurst index $\mathrm{H}=90$ shows that the stock has a fractal structure in $(0,1)$. In order to determine whether the stock has long-term memory, the $\mathrm{V}$ statistic is introduced.

The main purpose of $\mathrm{V}$ statistics calculation is to find the length of aperiodic cycle. The formula is as follows:

$$
\mathrm{V}=\frac{(\mathrm{R} / \mathrm{S})_{\mathrm{n}}}{\sqrt{\mathrm{n}}}
$$

The calculation of the $\mathrm{V}$ statistics using the raw data is shown in Fig. 1:

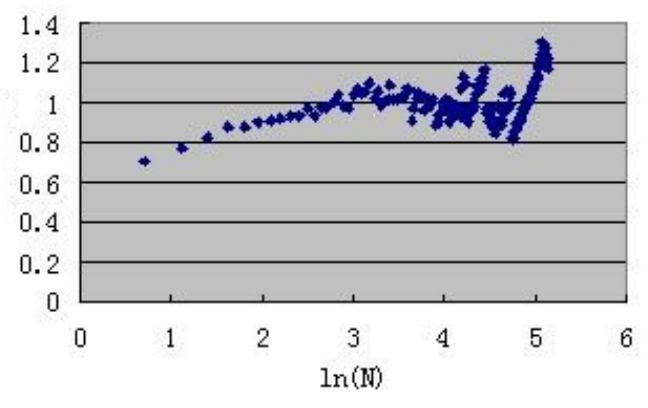

Fig.1. Value diagram of V statistics

In the value diagram of $\mathrm{V}$ statistics, it can be explained that if the line change of $\mathrm{V}$ statistics is horizontal, then the order is random. On the contrary, if there is an upward trend, the sequence is long-term memory. From the graph, it can see that the line of $\mathrm{V}$ statistic has a continuous upward trend, so the stock has long-term memory. The inflection point of the graph is the length of the sequence's aperiodic loop. The inflection point of the graph is the length of the aperiodic cycle of the sequence. The time series formed by the stock index is nonlinear, and the analysis results show that the stock has a 152-day cycle. The model and algorithm in this work are reasonable for the stock.

In model 1 and model 2, the initial price of stock is $\mathrm{S}(0)=46.79$, and the expected return $\mu$ is constant, equal to the bank interest rate $r=0.05$. The volatility of stock price $\sigma=0.2$ is estimated by SPSS software using statistical analysis method. Taking $\Delta t=4 / 1144$, the closing data of 1144 in four years is used for simulation. The intensity parameter of Poisson process is $\lambda=2$.

A simulation of stock prices using model 1 is shown in Fig. 2:

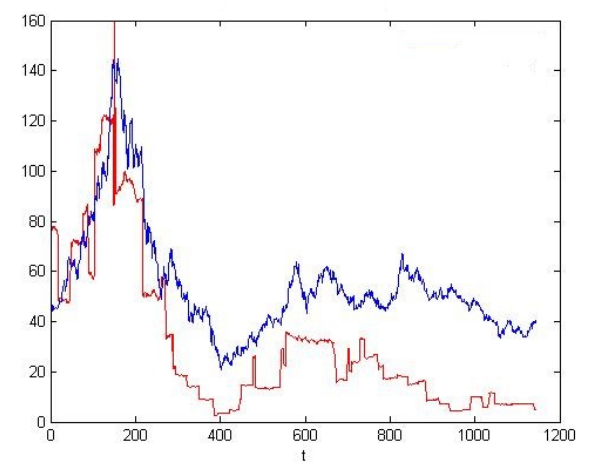

Fig.2. Model 1 comparison chart between stock price simulation and actual stock price

A simulation of stock prices using model 2 is shown in Fig. 3: 


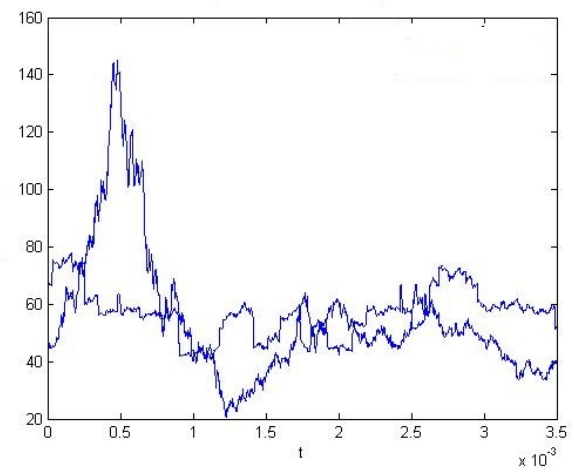

Fig.3. Model 2 comparison chart between stock price simulation and actual stock price

The comparison chart between model 1 and model 2 and the actual stock price movement is as follows:

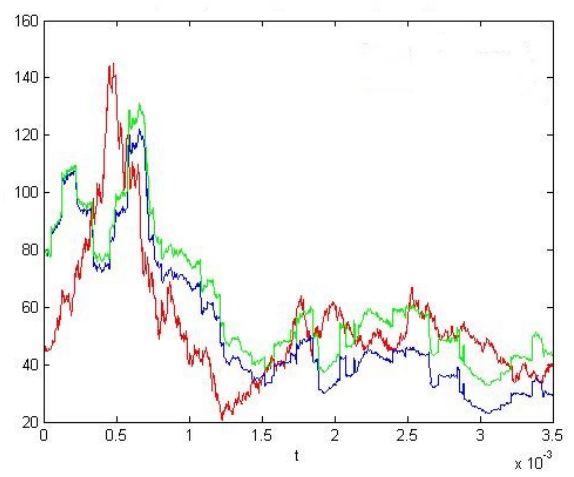

Fig.4. Comparison chart between model 1 and model 2 stock price simulation and actual stock price

\section{Result Analysis}

Model 1 and model 2 are simulated 1000 times under different parameters by MATLAB numerical method. The error control accuracy is calculated by the least square method, and the optimal simulation with accuracy of 0.623 and 0.542 is obtained, respectively. The simulation results show that the two models are effective simulations for the actual stock price. Under the condition that the volatility of the stock is controllable, the simulation converges very quickly and has high accuracy.

TABLE I. Comparison of accuracy and convergence speed between model 1 and model 2 under different parameters

\begin{tabular}{|c|c|c|c|c|c|c|c|}
\hline Model & $\boldsymbol{\mu}$ & $\boldsymbol{\sigma}$ & $\boldsymbol{\lambda}$ & $\mathbf{n}$ & time & $\max (\mathbf{e})$ & $\|\mathbf{e}\|_{2}$ \\
\hline 1 & 0.05 & 0.01 & 2 & 10000 & 0.43 & 0.22 & 0.623 \\
2 & 0.05 & 0.01 & 2 & 10000 & 0.92 & 0.17 & 0.542 \\
1 & 0.3 & 0.1 & 2 & 10000 & 0.83 & 0.25 & 0.716 \\
2 & 0.3 & 0.1 & 2 & 10000 & 0.70 & 0.32 & 0.828 \\
\hline
\end{tabular}

Among them, model stands for model 1, model 2. $\mu$ is the expected return of the stock. $\sigma$ is the volatility of the stock price. $\lambda$ is the Poisson distribution parameter. $\mathrm{n}$ is the number of cycles. Time is the program run time. $\max (\mathrm{e})$ is the maximum relative error. $\|\mathrm{e}\|_{2}$ is the two norms of error.

\section{CONCLUSION}

Based on the stock price obeying the fractional Brownian motion jump-diffusion process, this work improved the existing model. The model of fractional jump-diffusion process is modified from two aspects. Firstly, the strength of Poisson jump process is modified in the model, and the jump process is controlled by using the sequence of uniform distribution. A more general model is obtained than that of the general fractional jump-diffusion process, and the jump intensity is well controlled. Secondly, a generalized compensation Poisson process is introduced into the model, and the jump height is controlled (i.e., the intensity of the jump can be controlled, and the width of the jump can be controlled). In the discrete form, the Taylor expansion is proposed for the first time to calculate the control factor, which makes the numerical calculation efficient and fast. From the results of numerical analysis, the two models are closer to the actual change form of the stock than the general fractional jump-diffusion process model, especially when the early fluctuation of the stock is more frequent and the jump is large. When the sudden jump of an individual stock is not strong enough, the simulation result of model 1 is better, and the simulation effect of model 2 is better when the jump of individual stock is more frequent and the time is shorter. On the whole, the long-term prediction effect is better. For the special variation form, the simulation results are better than the general model. It is not difficult to find that when the expected rate $(\mu)$ of return and the volatility $(\sigma)$ of the stock price change greatly, the calculation accuracy of the model decreases and the stability of the model is general. It can't deal with the sudden rise with the expected rate of return and volatility, and it can deal with the original data in sections, which can achieve better results.

\section{REFERENCE}

[1] Merton R C. Continuous-time Finance [M]. Cambridge M A: Blackwell Publishers, 1990.

[2] Duncan, T. E., Y. Hu and B. Pasik-Duncan. Stochastic Calculus for Fractional Brownian motion, I. Theory, SIAMJ. Control Optim, 2000, (38): 582-612.

[3] Rogers, L.C.G., Arbitrage with Fractional Brownian Motion [J]. Mathematical Finance, 1997, 7: 95-105.

[4] Knut K, AASE. Contingent Claims Valuation When the Security Prices is Combination of an Ito Process and a Random Point Process [J]. Stochastic Processes and Their Applications, 1988, 28(20): 185-220. 
[7] Rong Jing, Ma Hui. Fractional Brownian Motion Jump-diffusion Process Model of Stock Price [J]. Modern Economic Information, 2016(08): 281.

[5] You Zuowei, Liu Shancun, Zhang Qiang. Research on Pricing Model of Convertible Bonds under Mixed Fractional Brownian Motion [J]. Systems Engineering-Theory \& Practice, 2017, 37(04): 843-854.

[6] Lin Hanyan. Pricing of American Two-valued Options under Fractional Brownian Motion Model [J]. Mathematics in Practice and Theory, 2018, 48(16): 291-296.

[8] Y in Z-M. New Method for Simulation of Fractional Brownian Motion [J]. Journal of Computational Physics, 1996, 127(1): 66-72. 